\title{
Understanding Trading Interactions and Behavior in Over-the-Counter Markets
}

\author{
Chi-Hung Chen \\ University of Maryland \\ cchen07@umd.edu
}

\author{
Louiga Raschid \\ University of Maryland \\ louiqa@umiacs.umd.edu
}

\author{
Jinming Xue \\ University of Maryland \\ jinming.xue@rhsmith.umd.edu
}

\begin{abstract}
This research applies machine learning methods, in particular probabilistic topic modeling, to understand patterns of interactions for Over-the-Counter (OTC) trading in corporate bonds. The interactions are between broker-dealers (dealers) and clients, or between dealers. From reports of dealer transactions, we create documents representing the daily activity of each dealer. This includes four types of dealer activities: Buy from / Sell to a client, and Buy from / Sell to another dealer. We use Latent Dirichlet Allocation (LDA) based topic models to identify communities of bonds that are bought or sold (co-traded) on the same day. Some communities reflect an industry sector, while others have a concentration of specific bonds. Several topics temporally align to notable financial events. We group dealers around topics to understand their interactions with clients and other dealers. We observe a range of interaction patterns that merit further study, including the centrality of some dealer(s) to some topics. This research illustrates that topic modeling / community detection can indeed provide insight into dealer behavior for OTC trades.
\end{abstract}

\section{Keywords}

FINRA TRACE; OTC markets; Latent Dirichlet allocation; financial communities.

\section{INTRODUCTION}

Over-the-counter (OTC) refers to the process of trading (buying and selling) securities that are not listed on a public exchange such as the New York Stock Exchange (NYSE). OTC trading is facilitated via a distributed network of broker-dealers (dealers), in contrast to an open centralized exchange. Dealers are supposed to provide liquidity to customers, i.e., purchase bonds that client wish to sell, and to stabilize prices. In contrast, in a centralized exchange, customers typically trade with each other (via a limit-order book).

Permission to make digital or hard copies of all or part of this work for personal or classroom use is granted without fee provided that copies are not made or distributed for profit or commercial advantage and that copies bear this notice and the full citation on the first page. Copyrights for components of this work owned by others than ACM must be honored. Abstracting with credit is permitted. To copy otherwise, or republish, to post on servers or to redistribute to lists, requires prior specific permission and/or a fee. Request permissions from permissions@acm.org.

DSMM'19, June 30, 2019, Amsterdam, Netherlands

(C) 2019 ACM. ISBN 978-1-4503-6823-0/19/06 . . \$15.00

DOI: https://doi.org/10.1145/3336499.3338004
A distinct feature of an OTC market, in contrast to a centralized change, is the near monopoly power of the dealers, and their responsibility to provide liquidity (cash) to customers. OTC trading has several well understood disadvantages. For example, pre-trade price transparency is a concern, since only the final traded prices are disclosed upon the completion of a transaction. Customers may have to contact multiple individual dealers to request quotes. OTC trading also cannot benefit from the wisdom of the crowds, in contrast to a centralized exchange.

Our dataset is the OTC market in US corporate bonds. Bonds are complex and diverse; for example, they vary on time to maturity, exposure to the term structure of interest rate risk, issuer specific credit risk, liquidity risk, etc. Unlike stocks, there is low volume in the bond market, i.e., bonds may be traded a few times in a day or month. Unlike the large pool of investors for centralized markets, a smaller number (several thousand) of sophisticated institutional investors dominate trading in the secondary corporate bond market.

The complex pool, low volume and the lack of transparency and information exchange in OTC markets increases the role and importance of the broker-dealers (dealers) and their client interactions. FINRA Trade Reporting and Compliance Engine (FINRA TRACE) is the key source of data for this market. Historically, FINRA TRACE did not report on the identity of dealers, thus removing the opportunity for research on dealer behavior. This policy was changed in 2017 to provide an academic version that provides anonymized dealer identity. We note that FINRA makes this data available for academic research.

Research in [7] analyzed dealer behavior in the OTC market covering US corporate bonds from 2005 to 2014. The majority of trades in corporate bonds are simple transactions: a dealer buys a bond from a client and sells the bond to another client. Dealers will typically avoid holding inventory and will try to sell bonds on the same day as they are purchased. Inter-dealer transactions that are from one dealer to another dealer are also reported. A typical interdealer broker facilitated transaction, which amounts to a significant proportion of large volume transactions are labeled a five-handed trades that include the following counterparties: the client seller, the dealer who buys from the client, an inter-dealer broker, a dealer who sells to another client, and the client who buys the bond.

Dealer interactions were characterized to reveal three types of dealers, namely core dealers, peripheral dealers and interdealer brokers; see Figure 1 for their interactions. Core deal- 
ers are the dominant players and their activity covers a large part of the monetary flow with client sellers / buyers. Core dealers also interact with other dealers. Peripheral dealers exhibit a lower volume of client-dealer transactions. They appear to have more interactions with other dealers. Finally, the interdealer brokers (IDBs) primarily interact with other brokers and do not directly engage with clients. Our proposed research will study dealer behavior at a greater level of granularity, i.e., with respect to topics (groups of bonds) and may provide additional insight into dealer behavior and impact on the OTC market.

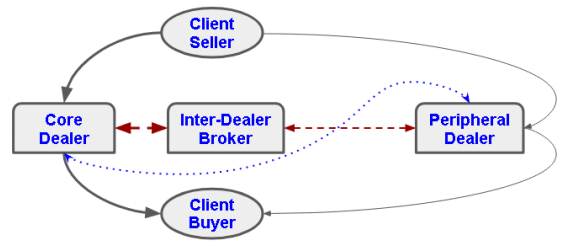

Figure 1: Illustration of Dealer Types and InterDealer Relationships in an Over The Counter (OTC) Bond Market (FINRA TRACE).

Research in [5, 4] used centrality metrics, e.g., eigen vector centrality, to compare more central dealers with peripheral dealers. A limitation of their research is that the centrality measures were computed over all transactions. They do not consider any heterogeneity of dealers' activities or specialization. For example, a central dealer may trade primarily in financial notes but rarely trade in debentures issued by utility companies. Our topic modeling research provides an approach to consider such features.

For our research, we use the reports of dealer transactions to create documents. Our research hypothesis is that daily trading activity is driven by clients' interests in buying or selling specific bonds or groups of bonds. For example, research in [2] suggests that multiple insurance companies may share some common bonds in their portfolios. When a disaster occurs and they need cash, they may all hold a fire sale of these common bonds. There may also be events involving specific companies, or specific industry sectors, that lead to trading activity across groups of bonds.

To summarize, we are interested in the co-trading (buying or selling) of groups of bonds, on each day. Hence, we identify a latent factor as a community of co-traded bonds. We are then interested in dealer behavior around these communities. This includes four types of dealer activities: Buy from / Sell to a client, and Buy from / Sell to another dealer.

We use Latent Dirichlet Allocation (LDA) based topic models [1] to identify communities of bonds that are cotraded on the same day. Some communities reflect an industry sector, while others concentrate on specific bonds. We are able to connect several topics to contemporaneous events, providing validation for the topics. We also observe varying temporal patterns for the topics, from continuous activity through the year, to local hotspots of point activity. Typically, the point activity is aligned with topic intensity. We note that the choice of the model was primarily due to its popularity with document collections. We plan to consider additional popular models including spectral clustering and tensor factorization.

We then group dealers around topics to understand their interactions with clients and other dealers. We focus on the most active dealers for each topic, for this initial study; most dealers are associated with one topic. One interaction pattern are dealers who buy from and sell to clients, creating a pattern of local trades. Another pattern are dealers who buy and sell from dealers (inter-dealer trades), with low levels of interaction with clients. We also observe dealers who engage in all four types of activity for a topic, buying from and selling to clients, and buying from and selling to dealers. There are also pass through dealers who primarily buy from a client and sell to dealers, or who primarily buy from a dealer and sell to a client. Finally, a topic may be associated with a mix of dealers exhibiting any of these types of interaction patterns. This research illustrates that topic modeling / community detection can indeed provide insight into dealer behavior for OTC trades.

\section{DATASET AND MODEL}

\subsection{Dataset}

Our dataset represents the Over-the-Counter (OTC) market in US corporate bonds. FINRA Trade Reporting and Compliance Engine (FINRA TRACE academic version) provides granular data at the level of the individual dealer. Each FINRA member (Broker Dealer Firms) has an obligation to report every transaction. TRACE includes the following items: date and time, the CUSIP (identifier of the bond), the trade price and volume, etc. The TRACE academic version includes the identity of the reporting party and the counterparty; the reporting party is a dealer while the counterparty may be a client or a dealer. Dealer identities are provided as an anonymized value, while there is no granular identification of clients.

TRACE data is available from $01 / 01 / 2005$ to $12 / 31 / 2014$. Following the protocol of [7], we focus on CUSIPs with FISD bond type of US Corporate Debentures and US Corporate Bank Note (CDEB or USBN). The Dick-Nielsen procedure [3] is used to delete cancellations and corrections. After applying some additional filters, the dataset includes 24.5 millions interdealer transactions and 37.7 millions client-dealer transactions. We consider the dataset for 2014 for this preliminary analysis; it includes over 6.5 million transactions, 12,077 CUSIPs and 1,282 dealers.

\subsection{Topic Models}

Topic models using the latent Dirichlet allocation (LDA) [1] have been used extensively for community detection. LDA is a generative probabilistic model for collections of discrete data (documents) that allows us to extract topics based on assumptions on the probability distributions. At a high level, it is assumed that to create a document, one first randomly chooses a distribution over a collection of topics. Then, for each word in the document, one independently and randomly chooses a topic from the previously sampled mix of topics assigned to the document, and then again independently and randomly draws a word from the word distribution for that topic. Based on these assumptions on the generative mechanism, topic models aim to extract the underlying topic structure via maximum likelihood estimation.

Following a topic modeling approach, there are multiple approaches to convert the stream of buy and sell orders into a document vector as follows: 
- Bond centric (Bc): Each document will represent the trading activity (each day) around a specific bond. The tokens (keywords) in each document will be selected from the set of Dealer IDs, and each document, identified by the Bond ID and Day, will represent the group of dealers who traded in that bond on each day.

- Dealer centric (Dc): Each document will represent the trading activity of each dealer (on each day). The tokens will be the Bond IDs and each document, identified by the Dealer ID and Day, will represent the group of bonds that are traded by that dealer on each day.

- Trade centric (Tc): Each document will represent the trading activity.The tokens will be the Bond IDs and each document, identified by the pair of Dealer IDs and Day, will represent the group of bonds that are traded between a pair of dealers on each day.

Our hypothesis is that a primary driver of trading activity are a group of bonds that clients wish to trade on a specific day. This results in a latent factor of a group of co-traded bonds forming a community. We thus chose the dealer centric approach to create documents, so that we can produce communities of co-traded bonds. We plan to compare this with other approaches to create documents.

To better understand individual dealer behavior, each trade is represented as both an activity for the buyer and the seller. When the trade is an inter-dealer trade, we have the identify of both dealers. However, a limitation of the dataset is that the clients who buy or sell bonds are not disaggregated. We could ignore these client transactions but this results in an unbalanced dataset, since the inter-dealer trades would be represented by two entries, versus one entry for a client buy or sell. To work around this problem, we created four types of documents for each dealer: BuyFromDealer, SellToDealer, BuyFromClient and SellToClient. For 2014, the dataset yielded 153,916 documents.

We used the Gensim LDA package [6]. For values of topic count ranging from 50 topics to 250 topics, the log-perplexity score ranged from 172 to 214 . While larger topic counts did indeed provide many very focused topics, it was also difficult to manually validate all of the topics. Thus, we report on the results for a topic count of 75 .

We note an important limitation of this first model is that all trades are considered equal. We do not use the nominal or face value of the bond, the price paid or trading volume, or the bond outstanding value (principal reduction) when creating the documents. As a result, we are not able to distinguish the market impact of a specific topic.

\section{SUMMARY OF RESULTS}

\subsection{Interpreting Topics}

We illustrate the topics using two exemplars, \#5 and \#46, in Figure 2. Each topic is characterized by the percentage of tokens, across all documents, that are included in the topic, as well as a set of tokens (identified by CUSIP). The blue shaded bar for each CUSIP reflects the percentage of tokens in the dataset that are covered by this CUSIP. The count on the $\mathrm{X}$ axis represents the count of occurrences of this token (CUSIP) in the dataset. The maroon shaded bar reflects the percentage of tokens of that CUSIP that are captured by this topic. The first topic relates to the (attempted) purchase of Times Warner Cable by Comcast. The second topic relates to the Petrobras scandal that involved prominent government personnel in Brasil; it includes bonds for multiple oil and energy related companies. Both are significant topics since they cover 1.1 to $1.7 \%$ of the tokens, respectively. Additional notable topics (not shown) include topic \#2 of multiple companies in the mining and energy sectors, including Molycorp, Taseko Mines, Peabody Energy and EV Energy Partners; all of these companies experienced bankruptcy or a significant drop in value in 2014 . Another topic \#61 includes several bonds issued by ADT; ADT acquired Reliance, a very large security company in Canada, in 2014.

We manually examined all of the topics; many appeared to reflect expected communities, e.g., bonds in the same industry sector. We plan to label these topics in future work, as will be discussed.

Figure 3 illustrates a temporal heatmap for selected topics. We observe a range of patterns: point activity, continuous activity across the interval and periodic or cyclic activity. For example, topics \#69 through \#75 have continuous activity through the year. The high intensity activity appears periodically in topic \#71. Topic \#16 has high intensity activity, through the year. Topics \#6 and \#13 have extended periods of high intensity over several weeks. Topics \#2, \#5, \#46 and \#61 have point activity. The point activity is notably temporally aligned with the real world events associated with the topics, e.g., the attempted purchase by Comcast in February 2014 (topic \#5).

\subsection{Dealer Association with Topics}

Our objective is to understand dealer interactions with clients and other dealers, around specific topics. This includes four types of dealer activities: Buy from / Sell to a client, and Buy from / Sell to another dealer. The topic model will assign each document with a vector of probability scores (topic weights) for each of the 75 topics. We consider the Top 3 topic weights for each document, and we also set a threshold of 0.1 , i.e., we do not consider topic weights below this threshold. Recall that each document is identified by the Dealer ID and Day, and represents the trading activity of each dealer. For each (dealer, topic) pair, we compute the daily average of topic weights, over all documents for that dealer; the average is computed over the 250 trading days for 2014 .

Table 1 reports on the distribution of dealers per topic, when we consider all edges. We also use a threshold for the daily average topic weight so that we can differentiate the significant dealers who are actively trading (throughout the year) on that topic. As can be observed, there is a very steep reduction in the count of dealer_to_topic edges, from over 56000 to 1344 , when we set a threshold of 0.05 ; a threshold of 0.2 retains only the most active dealers.

Figure 4 shows significant dealers (threshold $=0.2$ ) on the left associated with topics on the right. The color of the edge represents the transaction type. Green: Buy from client; Red: Sell to dealer; Pink: Buy from dealer; Blue: Sell to client. Our first observation is that most topics are associated with very few significant dealers; for threshold $=$ 0.2 , the range is from 1 dealer to 13 dealers. Most dealers are associated with one topic; for this threshold, only 8 dealers are associated with 2 topics. Topics \#6, \#44, \#46, \#49and \#65 are associated with multiple dealers. 


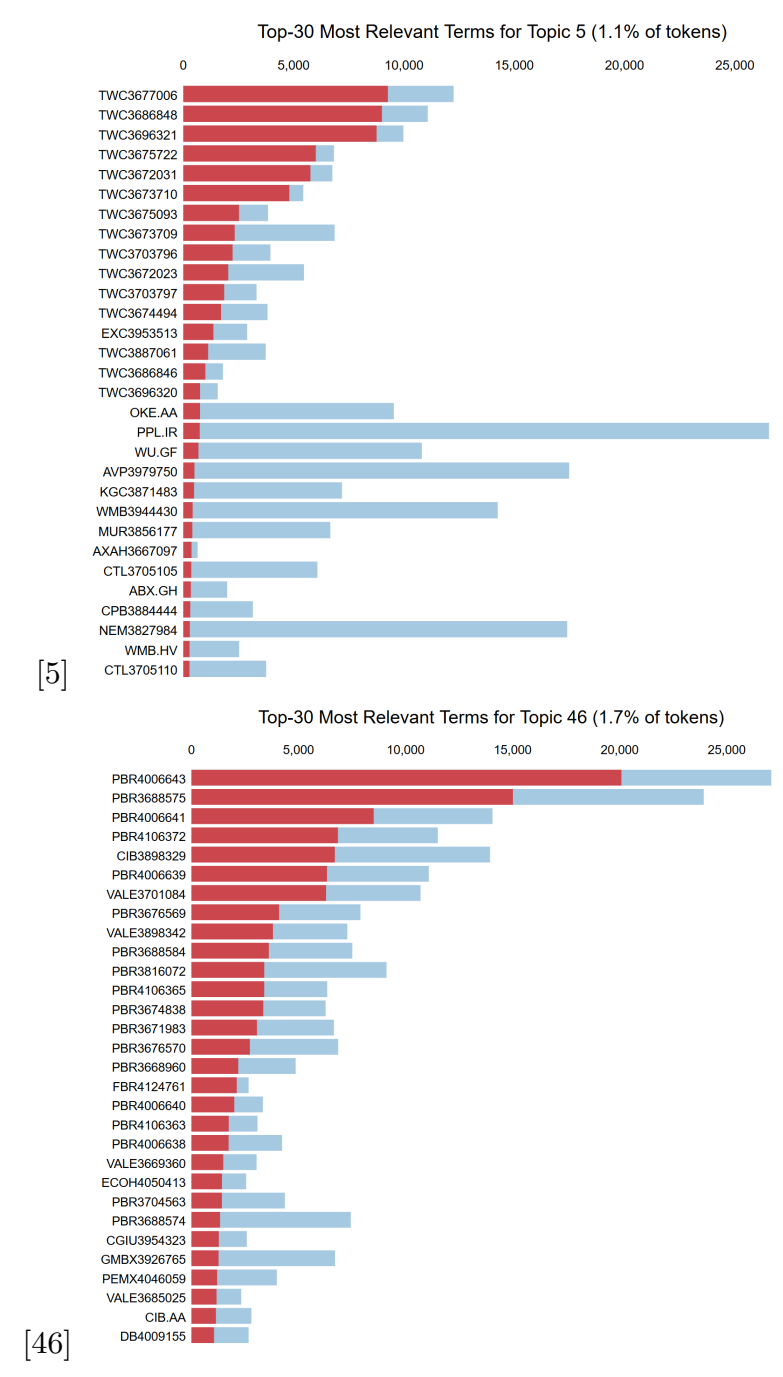

Figure 2: Attempted Purchase of Times Warner Cable by Comcast (February 2014) and the Brasil Petrobras Scandal (March 2014).

The significant dealer activity in topic \#44 is a combination of Pink: Buy from dealer and Blue: Sell to client; this is labeled as pass through trading activity. This suggests that purchases from clients for this topic are diffused over multiple dealers so that this activity is below the threshold for significance.

For topic \#65: we observe different dealer behavior. For example, 3 dealers have a combination of Red: Sell to dealer and Pink: Buy from dealer. These dealers show inter-dealer trades and do not have client interaction. There are also two dealers who are Green: Buy from client and one dealer who is Blue: Sell to client. For topic \#49, there are 4 dealers who display a combination of Green: Buy from client and Blue: Sell to client. These 4 dealers exhibit local trading with their own clients, without interacting with other dealers and their clients, for this topic.

Topics \#6 and \#16 appear to demonstrate the most diverse patterns. Topic \#6 has multiple dealers with the interdealer trade combination of Red: Sell to dealer and Pink: Buy from dealer. These dealers do not have client inter-

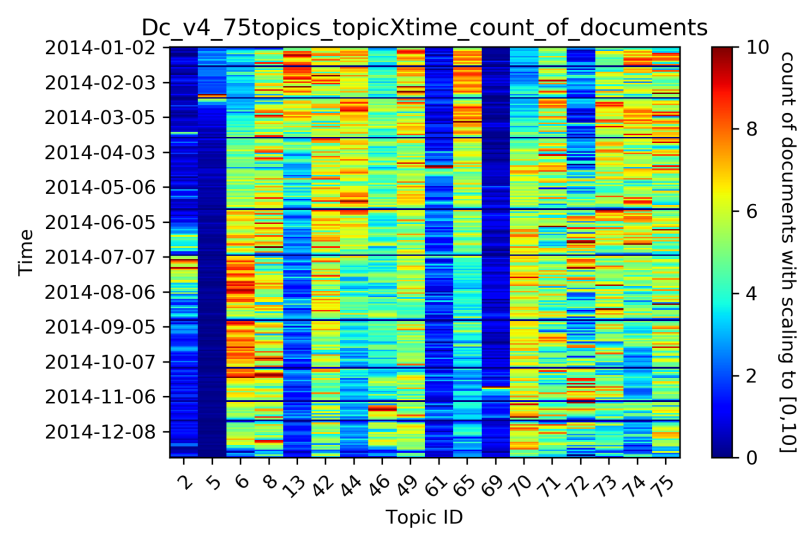

Figure 3: Temporal Evolution of Topics.

\begin{tabular}{|l|r|r|r|r|r|}
\hline \hline Threshold & $\begin{array}{r}\text { Topic } \\
\text { Count }\end{array}$ & $\begin{array}{r}\text { Edge } \\
\text { Count }\end{array}$ & MIN & MAX & AVG \\
\hline None & 75 & 56695 & 130 & 422 & 316.76 \\
0.05 & 66 & 1344 & 1 & 46 & 10.09 \\
0.10 & 53 & 591 & 1 & 27 & 5.64 \\
0.15 & 47 & 378 & 1 & 20 & 4.02 \\
0.2 & 39 & 254 & 1 & 13 & 3.3 \\
\hline \hline
\end{tabular}

Table 1: Count of Dealers per Topic for Different Activity Thresholds.

action. Topic \#16 has multiple dealers with a local trade combination of Green: Buy from client and Blue: Sell to client. This suggests that these dealers are trading locally with their own clients, and not interacting with other dealers. However, we also observe that both of these topics have multiple dealers who display a combination of edges of all four colors or activities. These dealers are thus trading locally with their own clients, as well as interacting with other dealers and their clients.

\subsection{Dealer Temporal Activity Around Topics}

From Figure 4, we observe that for this high significance threshold $=0.1$, most dealers are associated with one topic. There are a few exceptions. For example, dealer 1564 is significant in pass through trades of buying from clients and selling to other dealers for topic \#42. This dealer is also significant in pass through trades of buying from dealers and selling to clients for topic \#71.

Figures 5 and 6 drill deeper into dealer activity; we use dealer 2771 as an exemplar. We relax the threshold to show dealer activity over all 75 topics, even if the activity is low or non-existent. We also show the temporal patterns of dealer activity. Consider topic \#13. There is high intensity of this dealer buying from clients, in particular, during the first half of 2014. Similarly, there is high intensity of this dealer selling to clients, for this topic. The intensity of selling to dealers and buying from dealers is also continuous through 2014 , but at a lower intensity. This reflects a dealer who is active on this topic, both buying from clients and selling to clients. This dealer also sells and buys on this topic from other dealers, so we can conclude that the activity is not localized to her own clients.

In contrast, consider dealer 725; we do not include images due to limited space. This dealer shows symmetric behav- 


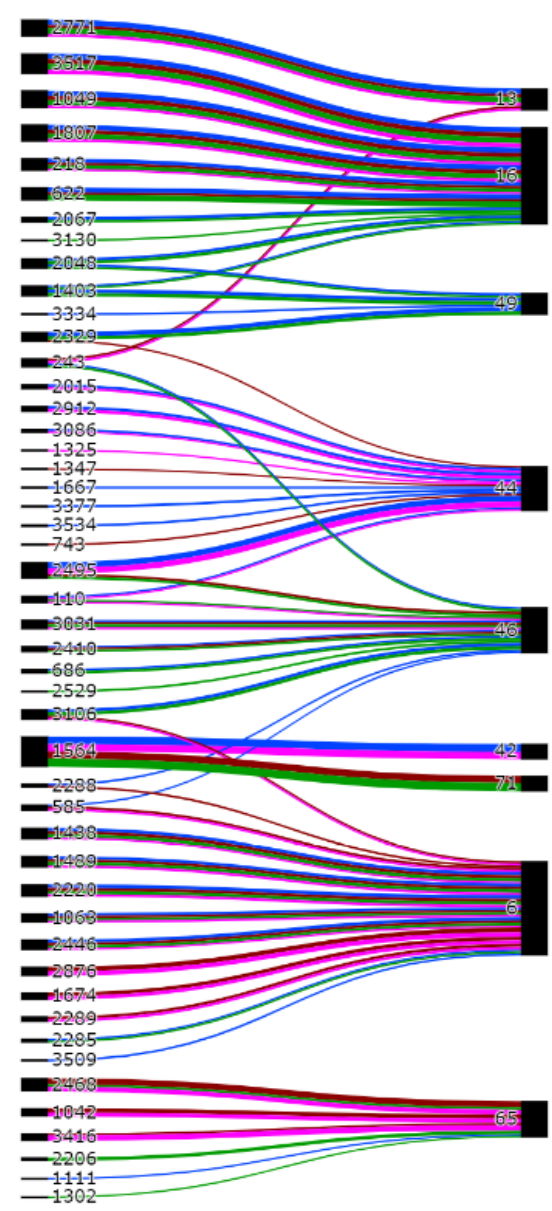

Figure 4: Communities with Dealers on the Left and Topics on the Right and 0.2 THRESHOLD for the most significant dealers. The color of the edge identifies the behavior. Green: Buy from client; Red: Sell to dealer; Pink: Buy from dealer; Blue: Sell to client.

ior for topic \#8, i.e., this dealer has significant activity in buying from clients and selling to dealers, as well as buying from dealers and selling to clients.

In such complex cases, these dealers may be exhibiting a mix of local, pass through and inter-dealer trading activity, over different intervals.

\section{CONCLUSION AND FUTURE WORK}

This research illustrates that topic modeling / community detection can indeed provide insight into dealer behavior for OTC trades. We consider the following in future researh:

- We will extend this preliminary model. We will consider alternate approaches to creating documents and we will also consider additional features of the trade such as price and volume. We will also compare topic model results with other popular models including spectral clustering and tensor factorization.

- We will label the topics so that they can be interpreted and validated. To do so we will consider the industry sector and ratings of the bonds. We may also differentiate smaller retail trades from institutional (high volume) trades. Finally we will compute useful economic metrics such as the average (weighted) return for the bonds in the topic, in comparison to the market return for all bonds.

- We will use topics, and communities of dealers around topics, to drill down into the dataset, to determine the impact of (topic) centrality on liquidity, across a range of topic characteristics. Research in $[5,4]$ explored the liquidity provided by dealers who are central or dominant in an OTC exchange. Such dealers are typically also the most active dealers, and there is often correlation between the level of activity and liquidity. It is therefore unclear if the liquidity that they provide is due to their centrality or their high level of activity, or a combination of these factors. Our research in contrast can identify communities of dealers around a topic; these dealers may not be dominant or may not be the most active dealers in the OTC exchange. This will provide more nuanced opportunities to study liquidity around topics and dealers.

\section{ACKNOWLEDGMENTS}

This research was partially supported by NSF grants CNS1305368 and DBI1147144, the Smith School of Business and a JP Morgan Chase AI Faculty Award.

\section{REFERENCES}

[1] D. M. Blei, A. Y. Ng, and M. I. Jordan. Latent dirichlet allocation. Journal of Machine Learning Research, 2003.

[2] M. Chaderina, A. Muermann, and C. Scheuch. The dark side of liquid bonds in fire sales. Available at SSRN: https://ssrn.com/abstract=2995544, 2018.

[3] J. Dick-Nielsen. How to clean enhanced trace data. Available at $S S R N$ : https://ssrn.com/abstract=2337908, 2014.

[4] M. DiMaggio, A. Kermani, and Z. Song. The value of trading relations in turbulent times. Journal of Financial Economics, 124:266-284, 2017.

[5] B. Hollifield, A. Neklyudov, and C. Spatt. Bid-Ask Spreads, Trading Networks, and the Pricing of Securitizations. The Review of Financial Studies, 30(9):3048-3085, 2017.

[6] R. Rehurek and P. Sojka. Software framework for topic modelling with large corpora. In Proceedings of the LREC 2010 Workshop on New Challenges for NLP Frameworks, pages 45-50, 2010.

[7] J. Xue. Two-sided otc dealer network. University of Maryland Smith School of Business Technical Report, 2018. 

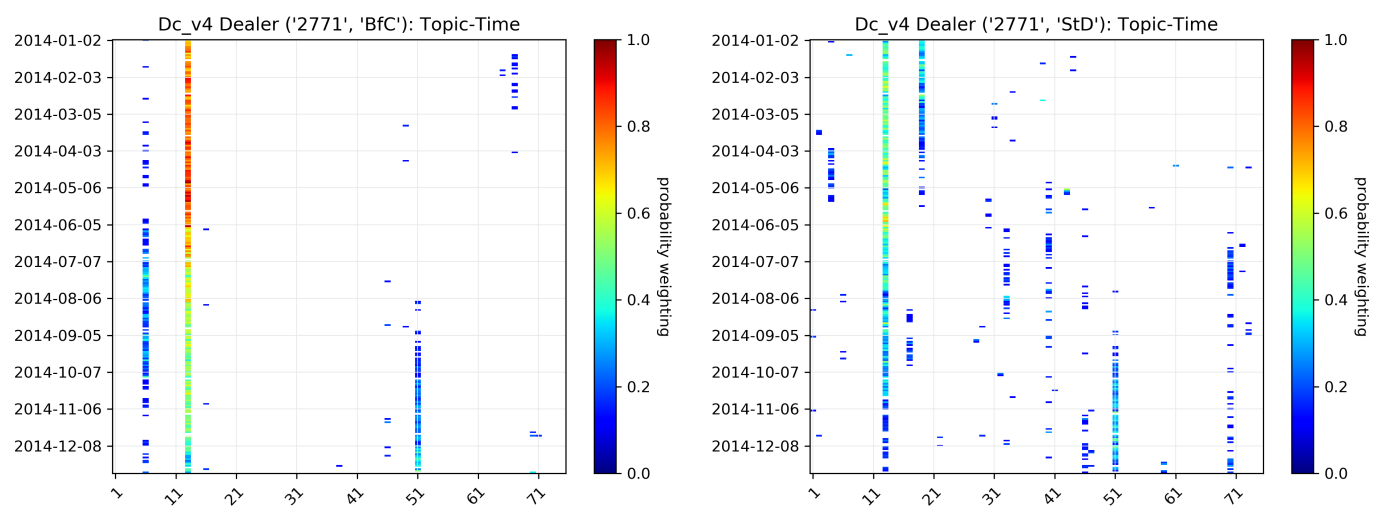

Figure 5: Temporal Activity Patterns for Dealer 2771 Buying from Clients and Selling to Dealers.
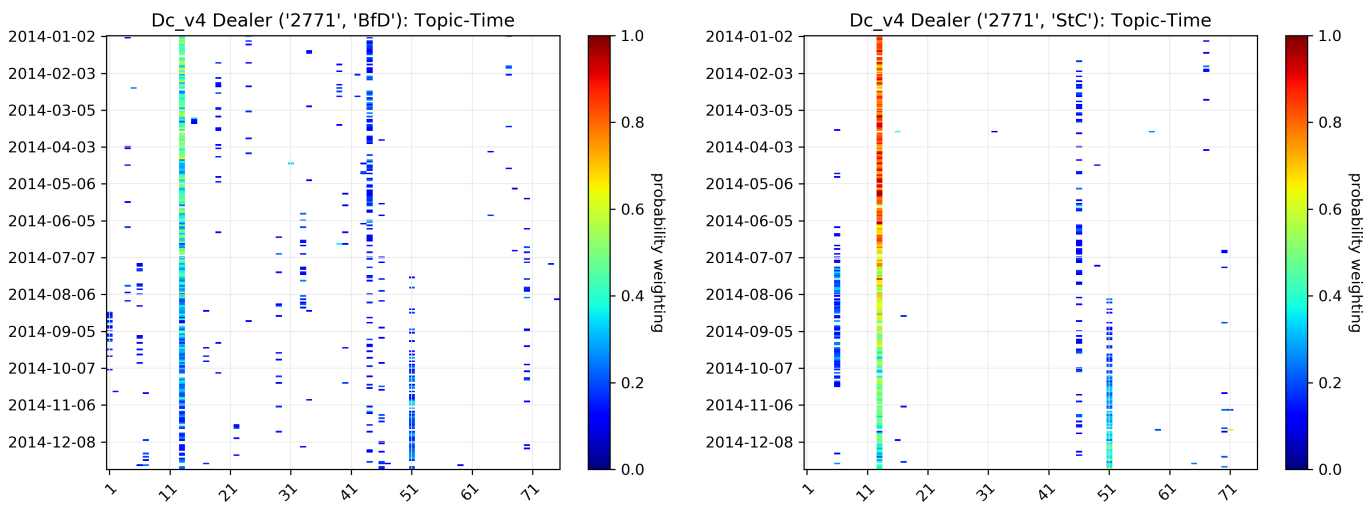

Figure 6: Temporal Activity Patterns for Dealer 2771 Buying from Dealers and Selling to Clients. 